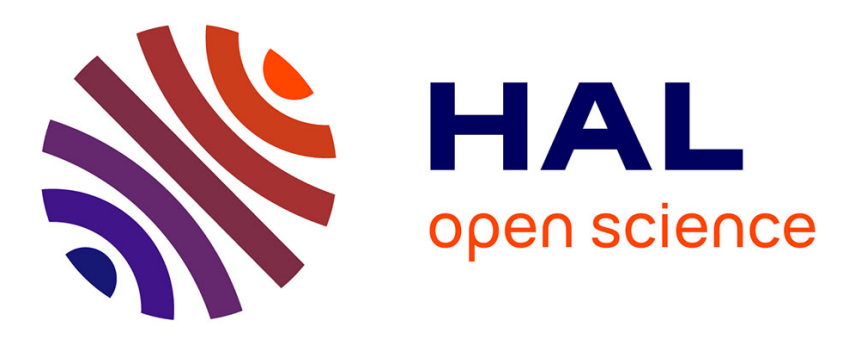

\title{
Deux échelons de citoyenneté? En quête de la citoyenneté archaïque
}

Alain Duplouy

\section{To cite this version:}

Alain Duplouy. Deux échelons de citoyenneté? En quête de la citoyenneté archaïque. Vincent Azoulay; Paulin Ismard. Clisthène et Lycurgue d'Athènes. Autour du politique dans la cité classique, 109, Editions de la Sorbonne, pp.89-106, 2011, Histoire ancienne et médiévale, 978-2-85944-682-6. hal-02404603

\section{HAL Id: hal-02404603 https://hal.science/hal-02404603}

Submitted on 18 Dec 2019

HAL is a multi-disciplinary open access archive for the deposit and dissemination of scientific research documents, whether they are published or not. The documents may come from teaching and research institutions in France or abroad, or from public or private research centers.
L'archive ouverte pluridisciplinaire HAL, est destinée au dépôt et à la diffusion de documents scientifiques de niveau recherche, publiés ou non, émanant des établissements d'enseignement et de recherche français ou étrangers, des laboratoires publics ou privés. 
Histoire ancienne et médiévale - 109

Université Paris 1 Panthéon-Sorbonne

\title{
CLISTHÈNE ET LYCURGUE D’ATHÈNES
}

\author{
AUTOUR DU POLITIQUE \\ DANS LA CITÉ CLASSIQUE
}

sous la direction de

Vincent Azoulay et Paulin Ismard

Ouvrage publié avec le concours

du Conseil scientifique de l'université Paris 1 Panthéon-Sorbonne

et de l'UMR ANHIMA (Anthropologie et histoire des mondes antiques)

Publications de la Sorbonne

2011 


\title{
Deux échelons de citoyenneté ? En quête de la citoyenneté archaïque
}

\author{
Alain DuPLOUY (université Paris 1 Panthéon-Sorbonne)
}

La structure institutionnelle et administrative de l'Athènes classique, héritée des réformes clisthéniennes, offrait aux citoyens athéniens une définition juridique claire de leur statut, garanti et contrôlé à travers divers organes et résumé par une double affiliation, à un dème et à une phratrie. Pour bien mesurer l'importance des réformes prêtées par la tradition à Clisthène, il conviendrait au départ de mieux connaître l'Athènes archaïque et, en particulier, la manière dont la citoyenneté était alors conçue. Les historiens ont en effet beaucoup plus de mal à définir ce même statut civique dans l'Athènes préclisthénienne. L'essentiel du problème vient notamment de la difficulté qu'il y a à s'abstraire du modèle et des concepts propres à l'époque classique pour penser les réalités archaïques.

En marge d'une recherche plus vaste sur la nature de la citoyenneté archaïque $^{1}$, je souhaiterais revenir ici sur l'une des définitions les plus courantes en la matière: le citoyen en tant que détenteur d'une autorité politique. De nombreux historiens évoquent en effet une distinction radicale, dans les cités de la Grèce archaïque et classique, entre deux catégories de membres du corps civique : les citoyens de "plein droit ", qui seuls disposaient de prérogatives politiques au sein des organes institutionnels de la cité, et les autres qui, bien que possédant le statut civique, ne participaient normalement pas à la vie politique, sinon en simples témoins. Cette distinction trouve des expressions diverses selon les auteurs: il est ainsi question de "citoyens complets" ou "actifs", de full citizens ou de vollberechtigte Bürger pour les uns, de "demi-citoyens", de "citoyens passifs", "diminués" voire "virtuels", de under-privileged citizens ou de Minderberechtete pour les autres. Dans tous les cas, il s'agit de marquer l'existence de deux échelons bien caractérisés dans le statut de citoyen. La distinction renvoie en fait à une opposition que formulait déjà Aristote entre deux catégories de politai, les archontes d'une part, les archomenoi de l'autre. L'idée est donc ancienne et a infiltré tout un pan de l'historiographie moderne, jusqu’à des études fort récentes.

\footnotetext{
${ }^{1}$ Voir Brock et DuplouY éd. (à paraître). Sur le sujet, voir notamment DaVIES 1977-1978; MANVILle 1990 ; RUZÉ 2003, p. 165-174 ; BLOK 2005; ISMARD 2010.
} 


\section{Esquisse antique et définition moderne de la citoyenneté de plein droit}

S'attachant à une étude des formes de constitution envisageables dans les cités grecques, Aristote entame le livre III de la Politique par une interrogation liminaire sur le citoyen et sur les principes régissant le concept de citoyenneté 2 . Le Stagirite relève tout d'abord la variabilité des conceptions effectives en relation avec sa propre taxinomie des régimes grecs : qui est citoyen dans une démocratie souvent ne le sera pas dans une oligarchie. Il écarte ensuite les critères insatisfaisants que sont pour lui le lieu de résidence ou le droit d'ester en justice, qui sont également accessibles à des catégories d'individus ne possédant pas le droit de cité. Enfin, Aristote se débarrasse des faux problèmes que sont les enfants, trop jeunes pour être inscrits, les " citoyens émérites» que sont les hommes libérés de leurs obligations militaires, ou bien encore les individus frappés d'atimie ou d'exil. Le citoyen au sens strict $(\dot{\alpha} \pi \lambda \tilde{\omega} \varsigma \pi 0 \lambda i \tau \eta \zeta)$ se caractérise par sa participation à la justice et à

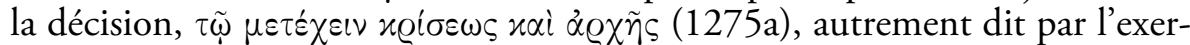
cice d'un pouvoir judiciaire et politique. Parmi les archai, Aristote inclut non seulement les magistratures limitées dans le temps, éventuellement renouvelables, mais surtout les fonctions à durée illimitée de juges et de

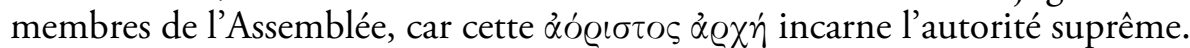
Sont dès lors citoyens, pour Aristote, ceux qui participent de la sorte au pouvoir.

La définition proposée s'applique avant tout, de l'aveu même d'Aristote, à une démocratie. Elle suppose donc une légère correction pour convenir à

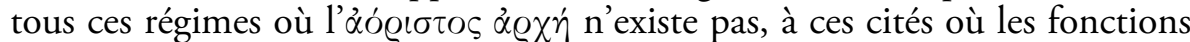
délibérative et judiciaire sont confiées à quelques individus, membres de conseils restreints ou magistrats spécialisés. Les citoyens seront donc ceux qui ont la possibilité de participer au pouvoir délibératif et judiciaire, même s'il est des moments où ils ne l'exercent pas. Le philosophe introduit ce faisant une distinction entre deux catégories de citoyens, le gouvernant

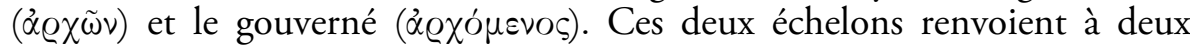

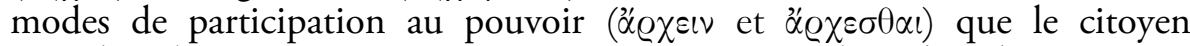
complet doit en principe expérimenter à tour de rôle. Ils renvoient

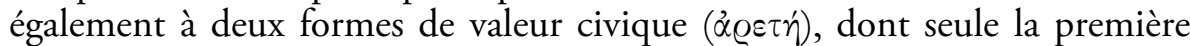
autorise les uns à commander aux autres. Et c'est là que se joue la distinction. Pour prendre un cas concret auquel se confronte Aristote, les artisans, qui n'ont aucune pratique de la vertu dans la vie quotidienne, peuvent-ils être citoyens? S'ils le sont effectivement dans certaines cités, c'est

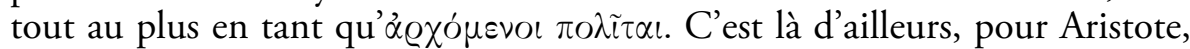
une tromperie, car cette citoyenneté de second ordre de ceux qui ne

\footnotetext{
${ }^{2}$ Aristote, Politique, III, 1-5, 1275a-1278a. Pour un commentaire détaillé, voir notamment MosSÉ 1967 et MosSÉ 1979b ; LÉVY 1980 ; JOHNSON 1984 ; OSTWALD 2000.
} 
participent pas aux honneurs s'assimile en fait au statut de métèque ( $ڤ ̋ \sigma \pi \varrho \varrho$

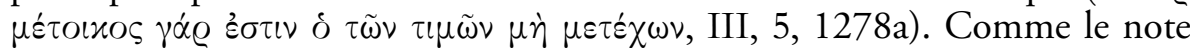
Edmond Lévy, il existe bien chez Aristote un « seuil qui sépare radicalement le citoyen complet de tous les autres ${ }^{3}$ ".

L'influence du schéma aristotélicien fut considérable dans l'historiographie contemporaine. Seule la Politique, qui fournit des définitions plus ou moins explicites, semblait en effet en mesure de pallier les carences de la documentation antique sur la notion de citoyen. Pareille approche de la citoyenneté grecque a donc naturellement trouvé un écho dans les études de droit public antique, au sein d'une perspective de recherche que l'on a récemment qualifiée de "mommsenienne ${ }^{4}$ ». L'idée trouve en l'occurrence une place déterminante dans les Griechische Staatskunde des maitres allemands du début du XX ${ }^{\mathrm{e}}$ siècle. Suivant les principes aristotéliciens, Georg Busolt systématise l'analyse du Stagirite: après un exposé théorique, l'historien passe à une série d'exemples concrets ${ }^{5}$. Dans les régimes oligarchiques, les citoyens de plein droit (Vollbürger) ne constituent qu'une fraction du corps civique. Les autres, bien que possédant les mêmes droits privés, n’avaient pas part aux affaires de l'État. D'un point de vue historique, plus le principe démocratique progressa, plus la citoyenneté de plein droit en vint progressivement à correspondre à l'ensemble des citoyens: Die Verbreitung des minderechtigten Bürgertums wurde immer weiter eingeschränkt, je ausgedehntere Geltung das demokratische Prinzip erlangte 6 . Inversement, la tyrannie se caractérise par une restriction extrême du plein droit de cité, tous les citoyens étant désormais soumis au tyran-alle Bürger ware ihre Untertanen ${ }^{7}$. Les changements de régime (metabolai) et la marche vers la démocratie, qui constituent souvent pour la pensée politique antique le sens de l'histoire, étaient en somme traduits chez Busolt en termes juridiques et appliqués au droit des personnes. Victor Ehrenberg n'en pense pas moins, lorsqu'il écrit - dans la version française de son célèbre ouvrage : "S'il a fallu distinguer ainsi non seulement entre citoyens et non-citoyens, mais aussi entre citoyens de plein droit et citoyens de droits restreints, c'est-à-dire entre gouvernants et gouvernés, cela exprime le fait très général que toute communauté implique l'exercice d'un pouvoir ${ }^{8}$."

Une analyse très semblable était menée simultanément en France par Gustave Glotz. Chez le savant français, la priorité ne va cependant pas au droit des personnes, mais bien à l'histoire politique des cités grecques.

\footnotetext{
${ }^{3}$ LÉVY 1980, p. 236.

${ }^{4}$ AZOUlay et IsMard 2007, p. 273.

${ }^{5}$ BusOlt 1920, $\$ 37$ (Das Bürgerrecht) et $\$ 48$ (Die Oligarchie des Adels und die der Begüterten).

${ }^{6}$ Busolt 1920, p. 221.

${ }^{7}$ BusOlt 1920, p. 223.

${ }^{8}$ EHRENBERG 1976, p. 78-84 (citation p. 84).
} 
L'auteur envisage en effet successivement la "Cité aristocratique ", la "Cité démocratique " et la "Cité au déclin ", selon un schéma censé traduire l' "évolution naturelle des institutions ». Pour Glotz, les instances de la cité démocratique ne se distinguent pas fondamentalement des organes des régimes aristocratiques ou oligarchiques : " À considérer les choses d'une façon superficielle, écrit-il, il n’y a de différences entre les deux régimes que pour le nombre de ceux qui en ont les bénéfices. Dans l'un et l'autre, les citoyens de plein droit peuvent assister à l'Assemblée, siéger au Conseil, être choisis comme magistrats. Mais, dès qu'on y regarde d'un peu près, on voit que la question du nombre est ici de telle importance qu'elle donne à des institutions d'apparence identique un caractère au fond tout différent ${ }^{9}$." Pour Glotz, la différence essentielle entre démocratie et oligarchie tient donc à la proportion de citoyens pouvant exercer de plein droit leur rôle politique : "Entre la démocratie et l'oligarchie, la différence essentielle consistait en ceci : dans l'une, tous les nationaux étaient citoyens de plein droit; dans l'autre, les citoyens de plein droit se distinguaient des citoyens de nature. L'oligarchie suppose toujours la division des nationaux en deux classes, dont l'une seule participe au gouvernement ${ }^{10}$. " C'était là une manière de projeter sur la cité archaïque, alors bien mal connue, les institutions de la cité classique, tout en ménageant une variable d'ajustement, en l'occurrence la notion de pleine citoyenneté et son extension.

C'est dans une perspective analogue que s'inscrivent les premiers travaux de Claude Mossé, en particulier son ouvrage sur Les institutions grecques à l'époque classique, qui a connu de multiples rééditions de 1967 à aujourd'hui. Dans l'économie générale du livre, l'histoire politique de l'époque archaïque est assimilée à un "élargissement du corps civique ou, plus justement, du corps politique ", c'est-à-dire du nombre de citoyens complets. La diversité des constitutions oligarchiques y est ainsi pensée en relation avec les modalités d'accès au corps des citoyens de plein droit.

Ajoutons que pareille distinction fut longtemps encouragée par un parallèle avec Rome, où l'existence d'une frontière entre un groupe social exemplaire, celui des honestiores, et le reste des citoyens - des rangs desquels pouvaient éventuellement émerger des homines novi- est parfaitement claire $^{11}$.

L'un des enjeux de cette approche juridique et institutionnelle de la citoyenneté était d'offrir des contours plus précis aux catégories sociales dominantes, sur lesquelles reposait l'exercice de l'autorité politique dans les régimes oligarchiques. Or, à l'exception de quelques réflexions d'Hippodamos de Milet (apud Aristote, Politique, II, 8, 1267b-1268a), de Platon (Timée,

\footnotetext{
${ }^{9}$ GLOTZ 1928, p. 92.

${ }^{10}$ GLOTZ 1928, p. 78.

${ }^{11}$ On verra sur ce point les remarques de HAMON 2007, particulièrement p. 86-87 et p. 99-100.
} 
24A-C ; Critias, 110C-112E) et d'Aristote lui-même (Politique, IV, 3-10, 1289b-1295a), les Anciens n'ont guère développé de pensée sociale en relation avec la définition des divers régimes politiques. La plus ancienne conception de la noblesse grecque ne remonte pas au-delà du XIX $X^{e}$ siècle. Comme chacun le sait, c'est à Numa Denys Fustel de Coulanges et à Eduard Meyer que nous devons la théorisation d'une telle structure sociale dominante ${ }^{12}$. La noblesse archaïque était alors perçue à travers l'organe censé en constituer la structure portante : le genos. Il ne s'agit pas de revenir ici, une énième fois, sur la mise en place et la déconstruction de cette chimère historiographique ${ }^{13}$. Il s'agit en revanche d'insister sur le caractère allogène de tout l'édifice social imaginé au $\mathrm{XIX}^{\mathrm{e}}$ siècle. Certes, les modèles des deux historiens sont bien différents, mais ils renvoient tout de même l'un et l'autre à des structures non grecques et non civiques. Fustel de Coulanges prêtait ainsi au genos une constitution et une évolution largement calquées sur la gens romaine, tandis que Meyer prenait appui sur la seigneurie médiévale pour construire son État gentilice (Geschlechterstaat). Au demeurant, tant chez Fustel de Coulanges que chez Meyer, le genos est une structure précivique, un héritage de l'organisation familiale primitive ou du Moyen Âge grec, bref une survivance antépolitique dans le monde de la cité. L'origine étrangère et surtout l'ancienneté de cette noblesse archaïque ne devaient donc pas manquer de perturber ceux qui cherchaient à décrire le fonctionnement institutionnel des cités. Renvoyer à un ailleurs spatial et temporel la justification d'une domination politique au sein de la polis n'était assurément pas de manière à satisfaire les historiens de la cité grecque. Sans nécessairement remettre en question la structure nobiliaire de la société, il convenait à tout le moins d'offrir une définition juridique et institutionnelle aux catégories sociales censées dominer les organes de la cité grecque. De ce point de vue, le concept de citoyen de plein droit offrait une réponse bien plus appropriée aux juristes et aux historiens de la Grèce ancienne que les brumes de la préhistoire grecque. Le concept permettait du reste d'inscrire la définition des classes dominantes dans les principes mêmes de la pensée politique antique et, peut-être mieux que tout, de l'établir sur une distinction aristotélicienne. La noblesse des genê, dont Fustel de Coulanges avait tracé l'origine jusque dans le culte des ancêtres, était ainsi sortie de l'histoire des religions et récupérée par une historiographie institutionnelle appelée à un grand succès. Elle se perpétua en ce sens durant une bonne partie $\mathrm{du} X \mathrm{X}^{\mathrm{e}}$ siècle à travers les termes d'aristocrates ou d'oligarques, qui furent pour l'essentiel assimilés à des citoyens de plein droit.

Il faut pourtant rappeler que ces termes, dont l'étymologie est certes grecque, n'existaient pas chez les auteurs antiques et que c'est aux historiens modernes que revient la responsabilité de leur avoir donné un sens dans la cité

\footnotetext{
${ }^{12}$ Fustel De Coulanges 1864 (livre II, La famille, chap. X. La gens à Rome et en Grèce) ; MEYER 1893, p. 291-320.

${ }^{13}$ BOURRIOT 1976 et RousSEL 1976 s'en sont chargés de manière définitive.
} 
grecque. On sait du reste aujourd'hui que la prééminence sociale de certains individus ne passait pas nécessairement par le contrôle des organes institutionnels, ni dans l'Athènes classique ni a fortiori dans les cités archaïques, où l'institutionnalisation du politique était encore loin d'être achevée. Autrement dit, toute conception de la hiérarchie sociale reposant sur la notion de pouvoir - entendu au sens d'exercice d'une autorité politique - s'avère en réalité bien peu opératoire à décrire les élites archaïques et classiques. Tout ou partie du problème de définition des élites grecques se résume en fait, on le sait, à la difficulté qu'ont eue les historiens contemporains à penser le prestige social sans nécessairement y associer le pouvoir politique ${ }^{14}$.

\section{Une nouvelle orthodoxie institutionnelle}

Si la construction théorique d'un Glotz ou d'un Busolt n'est plus que rarement reproduite en l'état, la notion de citoyen de plein droit n'en reste pas moins à la mode dans certaines études ${ }^{15}$. D'autant plus que la distinction entre deux échelons de citoyenneté bien caractérisés a reçu l'appui d'une nouvelle orthodoxie, celle du Copenhagen Polis Centre.

S'appuyant sur une analyse extrêmement détaillée de la mécanique institutionnelle athénienne de l'époque classique, Mogens $\mathrm{H}$. Hansen a progressivement étendu son enquête à la notion même de cité-État, pour fournir un inventaire des 1035 poleis archaïques et classiques. L'une des principales caractéristiques de l'approche danoise est de privilégier une définition institutionnelle de la cité grecque, au détriment bien souvent des rapports sociaux ou du cadre matériel dans lequel vivaient les Grecs. De fait, la réflexion du savant danois s'inscrit explicitement contre une conception holiste, durkheimienne, de la société, où les cultes - notamment - jouent un rôle fondamental dans l'organisation et la structuration de la communauté. Pour Hansen en revanche, il existe une césure claire entre le politique et un religieux qui n'est pas organisé institutionnellement. L'enracinement dans le droit et les institutions fournit dès lors une base beaucoup plus solide à la définition de la cité-État et, partant, du citoyen. En ce sens, Hansen a redynamisé la vieille approche juridique de la cité grecque proposée par Busolt ou Aristote. Et de fait, l'exégèse de la Politique offre des réponses à la plupart des questions que pose le Copenhagen Polis Centre.

Comme le philosophe antique, l'historien danois s'intéresse à la définition du citoyen et réaffirme avec force la distinction entre deux échelons de citoyenneté dans les termes mêmes où Aristote les présentait: All the ancient sources show that even in democratic poleis there was a clear distinction between

\footnotetext{
${ }^{14}$ Pour une discussion complète de l'approche politique des élites grecques, voir DuPLOUY 2005 et, pour une approche comportementale des statuts sociaux, DUPLOUY 2006.

${ }^{15}$ C'est le cas notamment de Welwei 1998 ou de Pomeroy, Burstein, Donlan et ROBERTS 2004, p. 70.
} 
archontes and archomenoi ${ }^{16}$. C'est aussi l'occasion pour Hansen d'insister sur le terme politeuma, qu'Aristote utilise, remarque-t-il, à dix-neuf reprises : In one case it seems to denote the active citizens in a democracy, in all other cases the reference is to the body of full citizens in an aristocracy or an oligarchy, i.e. to the small group of politai who through virtue or wealth have the sole right to participate in politics ${ }^{17}$. Seuls les régimes démocratiques offrent en effet une adéquation parfaite entre l'ensemble des citoyens et le politeuma, entendu comme corps politique, tandis que les diverses formes d'oligarchie archaïques et classiques multiplient en revanche les distinctions de qualification entre les membres du corps civique.

Au-delà de cette définition, l'argument revient également dans le débat qui a opposé Mogens Hansen à Moshe Berent sur la nature de la polis grecque. Pour ce dernier ${ }^{18}$, la cité grecque est l'exemple d'une "société sans Etat " (stateless society), à savoir - pour faire bref - une communauté politique qui ne dispose pas de gouvernement institutionnalisé et centralisé administrant un territoire et sa population. Il ne s'agit pas ici de prendre parti dans cette querelle taxinomique, mais bien de souligner la place que tient la notion de citoyen dans l'argumentation. Pour réaffirmer la nature étatique de la polis grecque, ainsi que présentée dans une étude antérieure ${ }^{19}$, Hansen insiste notamment sur la définition du corps civique : In relation to the structure and function of the state [...] citizens fall into two opposed groups: rulers and ruled, the first being identified with the government and the second with the subjects. Et d'ajouter l'affirmation suivante, étayée de diverses références à la Politique d'Aristote: At any time there is a clear distinction between on the one hand the rulers, called archontes, who issue commands and are empowered to enforce their commands, and on the other hand the subjects, called archomenoi, who have to obey orders and can be punished for disobedience ${ }^{20}$.

Dans l'inventaire des cités grecques archaïques et classiques, les principales formes de constitutions occupent une place significative dans le raisonnement ${ }^{21}$. Certes, Hansen n'organise pas son inventaire en fonction d'un classement politique en quatre catégories (démocraties, oligarchies, tyrannies et aristocraties), analogue à celui prêté par Diogène Laërce $(V, 27)$ aux 158 constitutions aristotéliciennes ; l'inventaire des 1035 poleis est organisé selon un classement strictement géographique. Mais la caractérisation des régimes en place et leur évolution à travers le temps constituent une

\footnotetext{
${ }^{16}$ HANSEN 2003, particulièrement p. 265 (thèse ${ }^{\circ}$ 30).

${ }^{17}$ HANSEN 1994b, p. 93.

${ }^{18}$ BERENT 1996 et BERENT 2000.

${ }^{19}$ HANSEN 2001.

${ }^{20}$ HANSEN 2002, p. 27.

${ }^{21}$ HANSEN et NiELSEN éd. 2004, p. 80-86.
} 
rubrique incontournable des notices, chaque fois du moins que les sources fournissent le moindre indice. Tout cela s'accorde bien entendu avec une conception juridique et politique de la citoyenneté grecque.

\section{Des exemples en latence}

À côté d'une conception théorique, récemment réactivée par Hansen, nombre d'exemples concrets de cités oligarchiques demeurent en outre en latence, où la présence supposée d'un corps restreint de citoyens de plein droit n'a pas toujours trouvé d'autres formes d'expression dans la recherche récente. Ces exemples, empruntés à l'histoire de quelques cités grecques toujours les mêmes d'ailleurs -, constituent en ce sens une survivance d'un mode d'approche du politique qui puise ses racines dans la tradition aristotélicienne.

Le premier et principal exemple invoqué est bien entendu la cité de Sparte, présentée comme le paradigme de tous les régimes oligarchiques, comme le modèle vers lequel les oligarques de toute cité et de tout temps se tournaient. Sans évoquer le peu de prérogatives laissées à l'Assemblée du peuple face à la Gérousie et aux rois, ni même la position hégémonique des Spartiates par rapport aux Périèques au sein de l'État lacédémonien, c'est une division du corps civique spartiate lui-même qui est en cause. Sur le témoignage de quelques auteurs anciens, certains historiens ont en effet coutume d'opérer une distinction entre les Homoioi, seuls citoyens de plein droit caractérisés par la détention d'un klêros et par leur participation au sussition, et diverses catégories d'individus globalement désignés comme "Inférieurs» (Hupomeiones). On doit probablement y ranger des groupes aussi divers que les Spartiates déchus faute de pouvoir acquitter leur contribution au sussition, les lâches ayant fait défection au combat (tresantes), les enfants nés de l'union d'un Spartiate et d'une femme hilote ou périèque (mothakes), les fils illégitimes (nothoi), les jeunes étrangers admis à l'agôgê (trophimoi) et les hilotes affranchis enrôlés dans l'armée (neodamodeis). Les critères d'exclusion du groupe privilégié des Homoioi étant en fait nombreux, les Spartiates auraient d'ailleurs laissé s'accroître, avec le temps, le nombre de ces inférieurs libres ${ }^{22}$. Comme le souligne Françoise Ruzé, "si, selon la tradition antique, cette cité était à dominante oligarchique, c'est qu'il y avait parmi les citoyens - les seuls qui comptent pour la définition d'un régime - un clivage entre ceux qui étaient écartés de toutes les magistratures, y compris l'éphorat, donc de toutes les initiatives politiques, et ceux qui pouvaient y avoir accès ${ }^{23} »$.

\footnotetext{
${ }^{22}$ Voir notamment SWOBODA 1926, \$70-72 (Die Rechtsstellung der verschiedenen Bevölkerungsklassen), particulièrement p. 659 (HAW IV/1/1/2) ; WELWEI 1998, p. 100-102 (Minderberechtigte Spartaner).

${ }^{23}$ RUZÉ 2003, p. 103. Voir aussi RUZÉ 1997, p. 218-224.
} 
Depuis une trentaine d'années, le renouveau de l'historiographie spartiate a cependant fait évoluer de manière considérable notre perception. En l'occurrence, l'image traditionnelle du régime spartiate apparait comme un " mirage ", selon l'expression consacrée, qui reflète une réalité à la fois déformée et fantasmée. Celle-ci a pour l'essentiel été mise en forme à partir du $V^{\mathrm{e}}$ siècle en dehors de Sparte, en particulier par les Athéniens, à l'appui d'une vision idéologique et tendancieuse de systèmes politiques concurrents. Les Spartiates eux-mêmes ne sont d'ailleurs pas en reste et ont puissamment contribué à l'invention de leur propre histoire, en projetant certaines réformes en cours dans un passé censé les légitimer ${ }^{24}$. Si tout exposé systématique de la société spartiate archaïque est en conséquence hors d'atteinte, il n'en est pas moins possible de décortiquer certaines constructions et de jeter un éclairage original, délesté de toute perspective athénocentriste, sur les réalités spartiates.

Parmi les «Inférieurs », pensés comme des citoyens de droit restreint, les spécialistes de Sparte ont coutume de ranger des individus aux noms très divers : tresantes, mothakes, nothoi, trophimoi ou neodamodeis, qui renvoient d'ailleurs à des domaines aussi différents que la légitimité de la filiation ou la participation à l'armée civique et à l'éducation commune. Si les dénominations en question sont disséminées à travers toute la littérature antique, de Tyrtée à Plutarque, le terme générique d'hupomeiones, qui cristallise toute la réflexion et le statut même de ces individus, n'apparaît en revanche que dans le seul récit de la conspiration de Cinadon, telle qu'elle est relatée par Xénophon (Helléniques, III, 3, 4-11). L'épisode est célèbre; il se déroule peu après l'avènement d'Agésilas, soit vers 399-397. Dénoncé aux éphores par l'un de ses hommes de main, Cinadon ne faisait pas partie, nous dit-on, des Homoioi (oủ $\mu \dot{\varepsilon} v \tau o r ~ \tau \tilde{\omega} \nu$ ónoi $\omega \nu$ ) et déclarait être mû par la volonté de

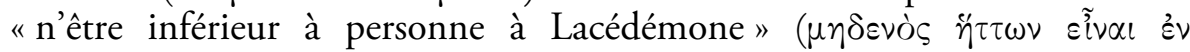
$\Lambda \alpha \varkappa \varepsilon \delta \alpha i \mu o v \imath$ ). Les conspirateurs n'étaient guère nombreux mais pensaient pouvoir compter sur l'appui des Hilotes, des Néodamodes, des Inférieurs et

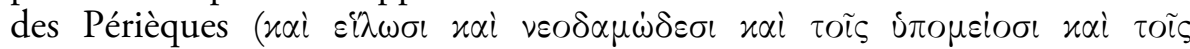
$\pi \varepsilon \varrho ı i x o ı \varsigma)$ pour renverser le régime des Spartiates et même, selon une formule homérique, les manger tout crus. Malgré son ampleur apparente, l'affaire se régla néanmoins promptement par l'arrestation de Cinadon et de ses complices, apparemment sans soulever le moindre mouvement social. Cet épisode, censé éclairer diverses distinctions sociales et politiques, n'en doit pas moins être replacé dans son contexte historique et littéraire. Paul Cartledge a montré que l'histoire de Cinadon avait tout d'abord pour fonction dans l'œuvre de Xénophon de révéler l'ampleur de l'oliganthropie, qu'il tient pour un mal structurel à Sparte. C'est d'ailleurs pour pallier le manque d'hommes que les Spartiates auraient enrôlé dans l'armée d'anciens

\footnotetext{
${ }^{24}$ Voir notamment HODKINSON et POWELL éd. 1994.
} 
hilotes; les néodamodes ne font en effet leur apparition dans nos sources qu'à la fin du V $V^{\mathrm{e}}$ siècle ${ }^{25}$.

Quoi qu'il en soit des causes profondes de l'oliganthropie spartiate, les interprétations ne manquent pas à propos des hupomeiones, sans qu'il soit nécessaire de recourir à la distinction aristotélicienne entre archontes et archomenoi politai. Pour Jean Ducat par exemple, ces individus ne constituent pas des « catégories stables, mais des groupes d'hommes en train d'effectuer un parcours social » descendant ou ascendant ${ }^{26}$. Ces diverses appellations renverraient donc à une dynamique sociale, qui n'appelle pas nécessairement une traduction institutionnelle et juridique. Jonathan Hall a pour sa part suggéré de lier ces désignations à deux systèmes d'affiliation civique distincts : l'une, horizontale, où chaque cité de Laconie (Sparte, Boia, Tainaros, etc.) possède ses propres institutions et citoyens; l'autre, verticale, relative à la Laconie dans son ensemble, dont tous les Lacédémoniens sont ressortissants. La reconnaissance d'une citoyenneté lacédémonienne distincte de la citoyenneté spartiate lui permet ainsi de mieux définir les groupes impliqués dans la conspiration de Cinadon. De ce point de vue, les Spartiates sont les seuls à posséder les deux citoyennetés, les néodamodes sont des hilotes récemment intégrés au corps des Lacédémoniens mais pas à celui des Spartiates, les hupomeiones sont des Spartiates déchus pour l'une ou l'autre raison (couardise, mixité du sang, etc.) mais qui conservent leur citoyenneté lacédémonienne, tandis que les périèques leur sont dans l'ensemble supérieurs car, en plus de la citoyenneté lacédémonienne, ils possèdent également le droit de cité dans leur propre $p o l i s^{27}$.

Ces deux interprétations ne sont pas nécessairement exclusives. Elles ont en outre l'avantage de sortir ces Inférieurs d'une opposition stricte avec les Homoioi au sein du corps civique spartiate. D'une part, la diversité des parcours individuels dans une société aux possibilités réelles de mobilité sociale - de bas en haut, comme de haut en bas de l'échelle sociale ${ }^{28}-$ interdit en pratique l'esquisse de catégories fixes, invariables juridiquement. D'autre part, l'imbrication de plusieurs systèmes d'affiliation civique ou, si l'on veut, identitaire à l'échelle de la Laconie permet de penser ces individus en relation avec les multiples communautés auxquelles ils appartenaient. En l'occurrence, la cité de Sparte et son corps civique n'étaient pas seuls en cause et il est inutile d'imaginer, en son sein, une quelconque distinction juridique entre citoyens de plein droit et inférieurs.

\footnotetext{
${ }^{25}$ CARTLEdge 1987, p. 164-165 ; CARTLEdge 2002, p. 263-270.

${ }^{26}$ DUCAT 1995, p. 100. Sur les voies de promotion des hilotes, voir DUCAT 1990, p. 155-173 (collaboration et promotion) et sur la catégorie bien floue en réalité des tresantes, DUCAT 2006.

${ }^{27}$ HALL 2000, particulièrement p. 80-81.

${ }^{28}$ Voir notamment HODKINSON 1983.
} 
Les critères d'exclusion particuliers régissant la définition du corps des Homoioi a souvent concédé à Sparte une place à part dans la description des régimes oligarchiques. Ailleurs, la restriction du nombre de citoyens de plein droit au sein du corps civique est normalement mise en relation avec les critères traditionnels de définition de l'aristocratie : le sang, l'argent et l'activité professionnelle.

Dans les régimes oligarchiques, la minorité dirigeante, celle qui possède toutes les prérogatives et obligations civiques, fut ainsi régulièrement définie par la noblesse de naissance et par la richesse. Avec le développement de l'industrie et du commerce dans le sillage des fondations coloniales, l'enrichissement des roturiers aurait en effet permis à l'argent de devenir le critère principal d'évaluation sociale et, partant, politique. "L'argent fait l'homme", déplorait Alcée (fr. 360 Voigt), et Théognis se lamentait de voir "l'argent abâtardir la race " (v. 190). La présentation est ancienne et renvoie, comme on le sait, à une vision gentilice et moderniste des élites et de leur rapport aux richesses, telles qu'elles étaient conçues dans la première moitié du $\mathrm{XX}^{\mathrm{e}}$ siècle $^{29}$. Dans cette même optique, la mise en place de catégories censitaires aurait permis aux nouveaux riches d'intégrer la frange des citoyens de plein droit, tout en laissant aux nobles leur prééminence dans un système où la richesse évaluée était avant tout foncière ou issue du travail de la terre ${ }^{30}$. Chez nombre d'historiens, la mise en place d'un système censitaire passe donc pour un élargissement du corps civique, quoique la disposition demeure à la base d'une conception oligarchique de la société.

De ce point de vue, à Athènes, le rôle et la place des thètes dans le système politique solonien ne sont pas clairs pour tous les historiens. Les thètes étaient-ils, en l'occurrence, des citoyens de plein droit? On peut en effet en douter dans la mesure où la Constitution des Athéniens $(7,3)$ précise qu'ils ne pouvaient accéder aux magistratures. Certes, leur participation à l'assemblée, garantie par le système censitaire lui-même, relève de l'aoristos archê aristotélicienne et devrait en principe suffire à leur reconnaitre le plein droit de cité. Claude Mossé fait néanmoins remarquer que les assemblées du dêmos ne devaient pas être très fréquentes et que, de toute façon, elles devaient se contenter d'approuver les décisions prises par le Conseil de l'Aréopage, au recrutement aristocratique ${ }^{31}$. De même, Kurt Raaflaub note que, à l'époque de Solon, les thètes were not excluded from the assembly, but they were not expected to speak or, I think, to vote $e^{32}$. Plus que la fonction politique réellement dévolue à ces individus, qui tient en ultime ressort à la nature reconnue à la

\footnotetext{
${ }^{29}$ Sur ce débat, voir DuPLOUY 2002.

${ }^{30}$ Un tel scénario se retrouve notamment chez STARR 1977.

${ }^{31}$ Mossé 1993, p. 23-24. Opinion analogue chez RHODES 1981, p. 141 : The lower-class men were expected [...] to attend as "brute votes" rather than active members.

32 RAAFLAUB 2006, p. 423.
} 
constitution solonienne - une démocratie censitaire ou une forme d'oligarchie?-, ces commentaires dénotent surtout le succès d'une approche juridique et politique de la notion de citoyen dans l'Athènes archaïque.

Autre motif récurrent de disqualification civique, une activité manuelle vile offre l'occasion à Aristote et à ses épigones de faire rentrer en ligne de compte divers exemples. Ainsi, selon le Stagirite (Politique, 1278a), à Thèbes, une loi écartait du gouvernement ( $\mu \dot{\eta} \mu \varepsilon \tau \varepsilon ́ \chi \varepsilon \iota \nu ~ \alpha \varrho \chi \tilde{\eta} \varsigma)$ quiconque n'avait pas cessé toute activité depuis dix ans. Toujours selon Aristote (Politique, VII, 12, 1331a), dans les cités thessaliennes, l'artisan, le paysan et le marchand ne pouvaient siéger à l'assemblée dont les réunions se tenaient sur l'agora. Dans l'ensemble, tous ceux qui avaient une activité manuelle ou commerciale étaient en somme écartés des magistratures et ne pouvaient souvent pas participer à l'Assemblée, quand ils n'étaient tout simplement pas exclus du droit de cité. C'est d'une certaine manière ce que laisse supposer Homère (Odyssée, XVII, v. 382-386), lui qui rangeait les démiurges parmi les étrangers. Et Platon (Lois, VIII, 848A) de préconiser qu'il en aille ainsi dans sa cité idéale. On sait aujourd'hui que cette disqualification civique, totale ou partielle, se justifie dans la pensée politique antique par une construction philosophique bien ancrée à l'époque classique, proclamant le mépris de toute activité manuelle. À cet égard, les historiens ont longtemps admis, à la suite de Jean-Pierre Vernant et de Moses Finley ${ }^{33}$, les préjugés antiques face au travail. Toutes les activités ne souffraient pourtant pas de la même dévalorisation et il importe de ne pas généraliser au concept lui-même de travail certaines critiques antiques. Des études récentes ont en effet fortement nuancé pareil jugement, notamment dans le domaine de l'agriculture ${ }^{34}$. Conseillant à son frère de travailler, Hésiode notait : "Il n'y a pas d'opprobre à travailler; l'opprobre est de ne rien faire. Si tu travailles, bientôt le paresseux enviera ta fortune : richesse toujours est suivie de mérite et de gloire" (Les travaux et les jours, v. 311-313). Qu'en aurait-il été de la définition du citoyen si Aristote avait adopté l'opinion d'Hésiode?

Il arrive enfin que le corps des citoyens de plein droit se trouve limité à un nombre déterminé de manière précise. C'est là l'occasion d'une dernière série d'exemples, inlassablement repris d'une étude à l'autre et d'ailleurs bien souvent empruntés à Aristote ${ }^{35}$. Outre les régimes des Cinq-Mille et des Trois-Mille à Athènes, dont nous reparlerons, font partie de cette catégorie les cités dont le corps politique - par opposition au corps civique dans son ensemble - semble avoir été limité à mille citoyens de plein droit. C'est le cas de Colophon, d'Oponte, de Kymè d'Éolide, de Crotone, de Rhégion et

\footnotetext{
${ }^{33}$ VERNANT 1965, p. 185-247 ; FINLEY 1984, p. 234-260.

${ }^{34}$ Sur le travail de la terre, AMOURETTI 1986, p. 214-216, ainsi que p. 239-244 sur le blocage des techniques antiques par mépris aristocratique pour le travail manuel.

${ }^{35}$ Ainsi, chez M. H. HANSEN, dans l'Inventory: Marseille $\left(n^{\circ} 3\right)$, Agrigente ( $\left.{ }^{\circ} 9\right)$, Crotone $\left(n^{\circ} 56\right)$, Rhégion $\left(n^{\circ} 68\right)$, Épidaure $\left(n^{\circ} 348\right)$, Héraclée du Pont $\left(n^{\circ} 715\right)$, etc.
} 
d'Agrigente, sans même compter les Six-Cents de Marseille et d'Héraclée du Pont ou les Cent-Quatre-Vingts d'Épidaure. In allen diesem Fällen, notait Georg Busolt, handelt es sich um die Zahl der berechtigten Bürger überhaupt et d'ajouter: je zahlreicher die bürgerliche Bevölkerung eines Staates war, desto exklusiver wirkte natürlich die Beschränkung der Vollberechtigung auf eintausend ${ }^{36}$. Défini comme un sous-ensemble du corps civique, le groupe privilégié des citoyens de plein droit donnait en effet au régime un caractère d'autant plus oligarchique que la simple citoyenneté était partagée par un nombre important d'individus.

Ces cités ne font pour l'essentiel l'objet que de brèves mentions chez les auteurs anciens, sans que le fonctionnement même du régime soit clairement expliqué. Les informations données sont à ce point laconiques qu'il paraît bien difficile de sortir de l'interprétation oligarchique antique et de poser le problème en d'autres termes que celui de la nature du régime.

Pourtant, les Mille de Colophon offrent un cas d'étude particulièrement éclairant, car il est possible de donner une substance plus concrète à ces prétendus "oligarques". Bien entendu, il est question chez Xénophane (fr. 3 West) d'individus vêtus de pourpre, arborant des chevelures apprêtées et exhalant l'odeur de parfums subtils. Tout ce luxe a longtemps conforté les exégètes modernes : un tel mode de vie aristocratique convenait parfaitement à la noblesse locale qui constituait elle-même une oligarchie restreinte de mille citoyens de plein droit. Et si le chiffre est élevé pour une petite cité d'Asie mineure, K.-W. Welwei notait: diese Ziffer ist indes nicht wörtlich $z u$ nehmen ${ }^{37}$. La situation est pourtant très claire. En effet, il est possible de suivre, tout au long des époques classique et hellénistique, l'histoire des Mille de Colophon à travers les actes officiels de la cité. Eberhard Ruschenbusch estimait qu'au milieu du $\mathrm{V}^{\mathrm{e}}$ siècle la taille du corps civique de Colophon, alors tributaire de la Ligue de Délos, était de 1200 citoyens $^{38}$. À la fin du IV siècle, une souscription publique destinée à financer la reconstruction de la muraille de la ville rassemble environ 850 citoyens, soit, selon les mots de Léopold Migeotte, "une grande partie du corps civique ${ }^{39}$ ». Mais surtout, une série ininterrompue de sept décrets hellénistiques des $\mathrm{III}^{\mathrm{e}}$ et $\mathrm{II}^{\mathrm{e}}$ siècles donnent le nombre de présents à l'assemblée, selon une formulation spécifique ( s'établit entre 903 et 1342 citoyens, soit " pas moins de mille en général », ce qui est exactement la formule employée par Xénophane au $\mathrm{VI}^{\mathrm{e}}$ siècle (oủ $\mu \varepsilon i o u s$

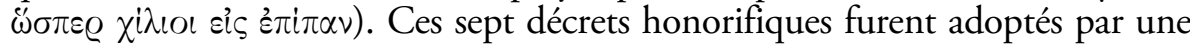
très large majorité des présents au terme d'une procédure de vote parfaitement

\footnotetext{
${ }^{36}$ BusOlT 1920, p. 354-358.

37 WelWeI 1998, p. 67.

${ }^{38}$ RUSCHENBUSCH 1983 ; RUSCHENBUSCH 1985.

${ }^{39}$ Migeotte 1992, p. 214-223 (n 69).
} 
démocratique, autrement dit secrète puisque réalisée au moyen de jetons. Selon Philippe Gauthier, ce chiffre de mille votants représente pour la cité hellénistique de Colophon la "totalité symbolique " de son corps civique ${ }^{40}$. Il faut donc souligner la récurrence de ce chiffre à Colophon de la période archaïque à la période hellénistique. Qui étaient dès lors les Mille de Colophon? Des oligarques? Des citoyens de plein droit? Je propose d'y voir tout simplement des citoyens, et même, plus exactement, la "totalité symbolique " du corps civique ${ }^{41}$. Rappelons du reste que, chez Xénophane, ces individus se rendent à l'agora ( montré Françoise Ruzé, doit à la fois être entendu comme le lieu de réunion et la réunion elle-même de l'ensemble des citoyens, bref l'assemblée ${ }^{42}$.

Un dernier exemple, emprunté à Benedetto Bravo, est peut-être encore plus troublant. L'historien italien a en effet suggéré que l'image habituelle des Gamoroi (ou Géomores) de Syracuse, celle d'une "élite oligarchique ou aristocratique au sein du corps civique, plus précisément un groupe de grands propriétaires fonciers détenant le privilège du pouvoir politique en tant que citoyens de plein droit (Vollbürger, comme le disent les savants de langue allemande) ", repose sur une vision "anachronique " et "modernisante" des réalités archaïques. Selon Bravo, au début du $\mathrm{V}^{\mathrm{e}}$ siècle, les Gamoroi syracusains, propriétaires terriens descendants des premiers colons, constituent ni plus ni moins que la "totalité des politai ", à savoir le corps civique - certes restreint - de Syracuse, par opposition aux esclaves bien entendu, mais aussi à une masse de libres non citoyens composant la majorité de la population libre de la cité. Ces derniers ne seraient donc en rien des " citoyens qui ne jouissaient pas de la plénitude des droits politiques, des citoyens exclus de la participation au pouvoir ", mais bien des individus à qui la citoyenneté (pure et simple) était déniée, c'est-à-dire en fait des "étrangers résidents ${ }^{43}$ ». Inscrite dans la série des exemples précédents, l'hypothèse de Bravo a tout pour plaire.

L'exemple des Mille de Colophon, celui des thètes athéniens et le cas des Inférieurs spartiates, pour ne pas parler des Gamoroi syracusains, nous montrent en somme qu'une approche juridique stricte des statuts civiques en fonction du classement des régimes antiques est particulièrement mal adaptée à la réalité de ces cités, tant à l'époque archaïque qu'à l'époque classique. La distinction stricte entre deux échelons de citoyenneté ne parvient pas à rendre compte de la structure sociale ni d'ailleurs de la nature même de la citoyenneté dans les poleis grecques. Devrions-nous dès lors remettre sur le métier les anciens paradigmes?

\footnotetext{
${ }^{40}$ GaUthier 1990 ; GaUthier 2003.

${ }^{41}$ Toute la démonstration en détail dans DuplouY (à paraître).

42 RUZÉ 1997, p. 25-28 ; RUZÉ 2003, p. 209-224.

${ }^{43}$ BRAVO 1992 (citations, passim).
} 


\section{Aux origines des catégories aristotéliciennes}

$\mathrm{Au}$ lieu de reproduire les catégories aristotéliciennes et d'inscrire toute réflexion sur la citoyenneté grecque dans ce cadre conceptuel, interrogeonsnous plutôt sur l'origine de la conception exposée pour la première fois par Aristote. Car il faut bien reconnaître que les exemples archaïques ou classiques censés justifier pareille distinction entre deux échelons de citoyenneté ne résistent guère, avons-nous vu, à une analyse nuancée des documents et ne peuvent donc, en eux-mêmes, être à l'origine des théorisations aristotéliciennes. Afin de mieux comprendre la construction aristotélicienne et, partant, de mieux critiquer son application rétrospective, il convient d'en retracer l'origine et d'identifier, autant que faire se peut, le contexte historique dans lequel elle a vu le jour.

C'est à Claude Mossé en particulier que l'on doit d'avoir parfaitement mis en lumière les ressorts historiques d'une telle définition de la citoyenneté grecque à l'époque classique. Le point crucial paraît bien avoir été les deux révolutions oligarchiques que connut Athènes à la fin du V siècle. Rappelons brièvement les faits.

En 411, les Quatre-Cents, qui s'étaient emparés du pouvoir à Athènes à la faveur de la débâcle de l'expédition de Sicile, promulguèrent une nouvelle constitution restreignant le corps civique. Outre le récit des événements chez Thucydide (VIII, 54-97), la Constitution des Athéniens $(29,5)$ précise que le nouveau régime réserverait la participation aux affaires publiques aux 5000 citoyens "les plus capables de servir par leurs biens et leurs personnes ", autrement dit aux hoplites; à charge pour les commissaires des dix tribus de dresser le catalogue de ceux-ci. On ne sait pas très bien quand et comment fut établie cette liste, qui comporta à un moment jusqu'à 9000 noms (Lysias, Pour Polystratos (20), 13). Quoi qu'il en soit, la nouvelle assemblée ainsi constituée ne se réunit en fait qu'entre l'expulsion des tyrans et le rétablissement de la démocratie quelques mois plus tard, à l'été de 410.

En 404, Lysandre, vainqueur à Aigos Potamoi, imposa à Athènes l'oligarchie des Trente, qui décida bientôt de dresser une liste de 3000 citoyens qui

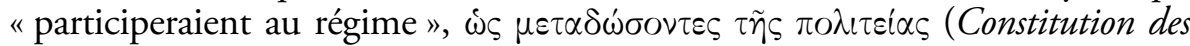
Athéniens, 36, 2). La liste du corps civique restreint voulu par les tyrans ne fut jamais rendue publique et fluctuait en fait avec l'humeur des Trente ${ }^{44}$. Critias fit néanmoins désarmer tous les Athéniens et les priva de toute garantie légale, en faisant ratifier par le Conseil une loi accordant les pleins pouvoirs aux Trente pour mettre à mort ceux qui ne faisaient pas partie des Trois Mille

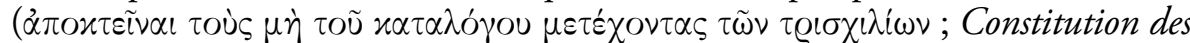
Athéniens, 37, 1). La majorité du corps civique était en ce sens mis hors la loi. Les dissensions entre les Trente précipitèrent cependant la chute du régime et le rétablissement définitif de la démocratie à Athènes en 403.

\footnotetext{
${ }^{44}$ Sur le modèle structurel du régime de 404, voir NÉMETH 2006.
} 
Les deux épisodes oligarchiques ne se prolongèrent pas suffisamment pour avoir des effets réels sur la composition du corps civique, mais Claude Mossé s'est souvent demandé ce qu'il serait advenu des Athéniens ne figurant pas sur la liste des Cinq-Mille ou des Trois-Mille en cas de succès durable de ces régimes ${ }^{45}$. Auraient-ils été tout simplement exclus de la citoyenneté, ou bien seulement écartés de toute activité politique et, partant, rangés dans une citoyenneté de second rang? Comme toute fiction historique, la réponse n'est pas simple. Elle conditionne toutefois la nature même de la citoyenneté que l'on reconnaîtra aux Cinq-Mille et aux Trois-Mille. Pour Lysias (Contre une proposition (34), 5), de telles dispositions - comme le décret proposé par Phormisios peu après le rétablissement de la démocratie en 403 - revenaient en fait à exclure purement et simplement du corps civique quantité

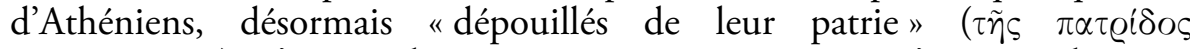

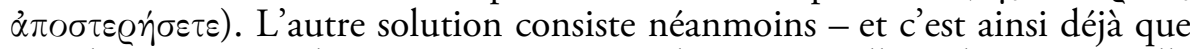
Busolt interprétait la situation ${ }^{46}$ - à tenir les Cinq-Mille et les Trois-Mille pour des citoyens de plein droit et à reléguer les autres dans un statut juridique inférieur, celui d'archomenoi politai.

Toute spéculation paraît vaine et, d'ailleurs, inutile. C'est là en effet une manière de poser le problème en des termes juridiques précis, alors qu'en cette fin de $V^{e}$ siècle le statut de citoyen n'était sans doute pas encore défini aussi strictement. Il est en revanche incontestable que les événements de 411 et de 404 ont grandement alimenté la réflexion politique au IV siècle et eurent, en ce sens, des répercussions théoriques plus importantes que les faits eux-mêmes. En l'occurrence, "faut-il supposer, notait Claude Mossé en 1967, que l'étude de certaines constitutions oligarchiques allait fournir au "parti oligarchique", qui dans l'Athènes du IV siècle se réduisait à un cercle restreint d'"intellectuels", la théorie de l'archomenos politês, formulée par Aristote $^{47}$ "? Quarante ans plus tard, l'hypothèse est toujours plus que séduisante. Rappelons d'ailleurs que dans la Constitution des Athéniens ellemême, le récit des deux révolutions oligarchiques se prolonge, sans solution

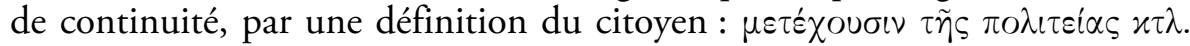
$(42,1)$.

Des deux formes d'exclusion - exclusion du corps civique ou exclusion du corps politique -, l'une était assurément moins radicale que l'autre et pouvait en ce sens être plus facilement défendue par des réformateurs modérés. C'est le cas notamment d'Isocrate. Loin d'être le pourfendeur de la démocratie athénienne, qu'il se défend d'ailleurs de vouloir remplacer par une oligarchie, Isocrate n'en fut pas moins l'un de ses critiques les plus

\footnotetext{
${ }^{45}$ Mossé 1962, p. 141-144 ; Mossé 1967, p. 20-21 ; Mossé 1979b, p. 244-247 ; Mossé 1993 , p. $70-71$.

${ }^{46}$ BusOlt 1920, p. 353-356.

${ }^{47}$ Mossé 1967, p. 21.
} 
acerbes $^{48}$. Ses discours, en particulier l'Aréopagitique, plaident en faveur d'une amélioration du régime existant. Il souhaite en fait un "retour " à la vieille démocratie de Solon, à cette patrios politeia équilibrée où les plus riches - qui sont en même temps les plus capables - dirigent les affaires de la cité sous le contrôle de la masse des citoyens. Renversant de manière habile les principes de l'oligarchie, il note, que dans un tel régime, les politiciens sont en fait les esclaves d'un maitre qui n'est autre que le dêmos. Il souligne ainsi les avantages d'une démocratie où la plupart des citoyens restent dans leur maison à la campagne et se rendent rarement en ville à l'assemblée ${ }^{49}$. Dès lors, tous les citoyens participent certes au contrôle judiciaire et à l'élection des magistrats - au demeurant bien préférable aux hasards du tirage au sort -, mais seuls les riches, qui ont tout le loisir nécessaire pour s'occuper des affaires de la cité, ont en réalité accès aux charges publiques. Bien qu'Isocrate n'en soit pas venu à ce degré de schématisation, l'idée d'une citoyenneté de second rang semble donc avoir fait son chemin dans certains cercles critiques de la démocratie. Aristote, dont l'œuvre politique marque l'aboutissement d'un siècle de réflexion en la matière, en vint quant à lui à formuler explicitement la distinction entre archontes et archomenoi politai.

La distinction aristotélicienne parut certainement d'autant plus opératoire que, en cette seconde moitié de IV siècle, la distance grandissait effectivement entre deux manières d'exercer sa citoyenneté, à tout le moins dans la présentation qu'en faisaient les orateurs, en particulier Démosthène ou Hypéride. Ceux-ci avaient en effet pris coutume de distinguer les politeuomenoi d'une part, ceux qui participent activement à la vie politique, autrement dit les "hommes politiques", et les idiôtai d'autre part, ceux qui se contentent d'écouter, bref la masse des "citoyens passifs ${ }^{50}$ ". Parallèlement, le terme politeuma, dont l'usage se diffuse précisément à partir de la deuxième moitié de IV siècle, semble désigner un corps restreint au sein de l'ensemble des citoyens, notamment chez Aristote ${ }^{51}$. La constitution de Cyrène (SEG 9.1) en offre, pour cette fin de IV siècle, un exemple épigraphique : la distinction y est faite entre le dêmos, entendu comme l'ensemble du corps civique, et un politeuma de dix mille, défini comme le groupe des citoyens actifs. Certes, la notion de politeuomenoi ne correspond pas exactement à la définition aristotélicienne du citoyen de plein droit, car cette "classe politique " n'était pas déterminée par la possession d'une charge officielle. Mais, dans les esprits, la voie était toute tracée pour préparer aux restrictions censitaires opérées par Antipatros en 322, puis par Cassandre en 317. L'époque hellénistique consacrerait ensuite cette distinction entre un

\footnotetext{
${ }^{48}$ Voir notamment OBER 1998, p. 248-289, particulièrement p. 277-286.

${ }^{49}$ Voir notamment Isocrate, Aréopagitique (7), 52.

${ }^{50}$ MosSÉ 1984.

${ }^{51}$ RUPPEL 1927.
} 
groupe de politeuomenoi et le reste des citoyens. Comme l'a montré Philippe Gauthier, il n'y a sur ce point guère de différences entre la démocratie athénienne $d u I V^{e}$ siècle et les démocraties des cités grecques de la haute époque hellénistique ${ }^{52}$.

Le concept de politeuma, la classe des politeuomenoi et la notion même d'archomenoi politai, qui sont forgés au cours du IV siècle, ont ainsi offert des cadres précis à l'aune desquels les cités grecques pouvaient être perçues. Ces repères, qui peuvent être utiles dans l'évaluation des régimes du second classicisme ou de la haute époque hellénistique, sont-ils pour autant applicables aux époques antérieures, et en particulier à l'époque archaïque, temps des oligarques s'il en est ? Autrement dit, ces catégories classificatoires issues de la réflexion, des expériences et de la pratique politiques de la fin du $V^{e}$ et du IV ${ }^{e}$ siècle justifient-elles l'existence, à toute époque et en toute cité, de deux échelons de citoyenneté ? Il faut sur ce point être très clair et reconnaître que l'application rétrospective de ce schéma fait en réalité fi des conditions historiques et intellectuelles de sa formulation au IV siècle. En plus d'avoir été forgée par la pensée classique, cette manière d'appréhender la citoyenneté ne convient à peu près bien qu'à l'Athènes démocratique du $\mathrm{IV}^{\mathrm{e}}$ siècle et aux démocraties de la haute époque hellénistique, mais s'applique particulièrement mal à tous les autres $\mathrm{cas}^{53}$. Vouloir, aujourd'hui, rendre compte de Sparte ou de Colophon dans les mêmes termes qu'Aristote, Busolt ou Hansen est fort peu opératoire. En réalité, le « citoyen de plein droit " et son corollaire le " citoyen passif " ont longtemps hypothéqué toute approche historique - et non théorique - de la citoyenneté. Ils ne sauraient en somme être en aucune manière des concepts utiles à une étude de la citoyenneté archaïque.

Le maintien par Mogens Hansen d'une approche juridique de la cité archaïque et classique témoigne tout au plus de l'impossibilité qu'ont certains savants contemporains à interroger la cité grecque dans des termes différents de ceux d'Aristote. En plus de fournir des réponses, la Politique suscite ainsi les questions elles-mêmes, comme si ce texte représentait l'alpha et l'oméga de toute approche de la cité grecque.

\footnotetext{
${ }^{52}$ Voir notamment GAUTHIER 1984.

${ }^{53}$ Pour une critique analogue de la pensée classificatoire aristotélicienne et de son application rétrospective aux systèmes archaïques, voir récemment BLOK 2005, p. 31-35.
} 